\section{Effect of oral fluticasone on refractory peptic esophageal stricture - a new therapeutic method}

Most benign esophageal strictures are a consequence of acid-induced mucosal injury [1]. The cornerstone of the management of benign strictures is still esophageal dilation [2]. A subgroup of strictures are refractory, and an alternative approach is required. Studies have shown prolonged benefit in terms of improving dysphagia and decreasing dilation frequency among patients receiving intralesional steroid injection [3-5].

However, some patients with refractory peptic esophageal stricture require frequent esophageal dilation, even with intralesional steroid injection. Intralesional steroid injection can only be performed at intervals, during endoscopy. We prescribed fluticasone inhalers (GlaxoSmithKline, Research Triangle Park, NC, USA) for patients. The inhalers were used without a spacer to deliver $50 \mu \mathrm{g}$ twice daily, after which patients were given water to aid in esophageal delivery of the steroid.

To avoid any possible bias, the oral steroid inhaler was given at alternate sessions of esophageal dilation with intralesional steroid injection (esophageal dilation with intralesional steroid injection was followed by esophageal dilation with intralesional steroid injection and the oral steroid inhaler, and so on). We analyzed the data from the first six sessions.
Four patients were enrolled. The mean age was 62 , and there were two women and two men ( $\bullet$ Table 1 ). Eosinophilic esophagitis was ruled out by multiple sessions of esophageal biopsies. All patients in this study received a proton pump inhibitor during the study. Use of inhaled oral fluticasone significantly decreased the frequency of esophageal dilations, more than did intralesional steroid injection ( $\bullet$ Table 2 and $\bullet$ Table 3). Esophagitis and esophageal stricture healed after several months of steroid inhaler therapy. There were no side effects with the fluticasone inhaler during this period.

To our knowledge, this is the first report of using oral steroid in the treatment of refractory peptic esophageal stricture. In the future, a multiple-center study is needed to study this novel observation further.

Endoscopy_UCTN_Code_TTT_1AO_2AH

Table 1 Details of patients treated for refractory peptic esophageal stricture.

\begin{tabular}{|c|c|c|c|c|}
\hline Parameter & Patient 1 & Patient 2 & Patient 3 & Patient 4 \\
\hline Age, years & 66 & 58 & 60 & 62 \\
\hline Gender & Female & Female & Male & Male \\
\hline History of GERD & Yes & Yes & Yes & Yes \\
\hline Other significant history & None & $\begin{array}{l}\text { Radiation therapy } \\
\text { for breast cancer }\end{array}$ & None & $\begin{array}{l}\text { Esophageal perforation } \\
\text { once after dilation }\end{array}$ \\
\hline Hiatal hernia & Yes & Yes & Yes & Yes \\
\hline Barrett's esophagus & No & No & Yes & Yes \\
\hline Daily dysphagia & Yes & Yes & Yes & Yes \\
\hline Esophagitis & Yes & Yes & Yes & Yes \\
\hline Transverse stricture without dilation & No & No & No & No \\
\hline Stricture location & Mid & Mid & Mid & Mid \\
\hline Stricture length & Short & Short & Short & Short \\
\hline
\end{tabular}

GERD, Gastroesophageal reflux disease.

Table 2 Days between esophageal dilations for patients treated for refractory peptic esophageal stricture with dilation only, dilation plus steroid injection, or dilation plus steroid injection plus oral steroid.

\begin{tabular}{|c|c|c|c|c|c|c|c|}
\hline \multirow[t]{2}{*}{ Therapeutic modality } & \multicolumn{5}{|c|}{ Time between esophageal dilations, days } & \multirow[t]{2}{*}{$P$ value } & \multirow{2}{*}{$\begin{array}{l}95 \% \text { confidence }^{1} \\
\text { interval }^{1}\end{array}$} \\
\hline & Patient 1 & Patient 2 & Patient 3 & Patient 4 & Mean \pm SD & & \\
\hline Dilation only & $35 \pm 7.2$ & $37 \pm 6.1$ & $44 \pm 5.2$ & $43 \pm 6.2$ & $39.42 \pm 6.9$ & - & - \\
\hline $\begin{array}{l}\text { Dilation plus intralesional } \\
\text { steroid injection }\end{array}$ & $62 \pm 5.8$ & $74 \pm 7.6$ & $68 \pm 8.0$ & $70 \pm 8.7$ & $68.41 \pm 8.2$ & $<0.001^{2}$ & $17.54-39.95$ \\
\hline $\begin{array}{l}\text { Dilation plus steroid } \\
\text { injection plus oral steroid }\end{array}$ & $118 \pm 16.1$ & $121 \pm 23.5$ & $106 \pm 15.8$ & $113 \pm 21.5$ & $115 \pm 17.6$ & $<0.001^{3}$ & $63.54-89.95$ \\
\hline
\end{tabular}

$195 \% \mathrm{Cl}$ of the mean difference in time between dilation-only intervals and the dilation plus further treatment intervals.

${ }^{2}$ Dilation only vs. Dilation plus intralesional steroid injection.

${ }^{3}$ Dilation only vs. Dilation plus intralesional injection and oral steroid inhaler.

Table 3 Days between esophageal dilations when patients were treated with dilation plus steroid injection plus one, two and three sessions of oral steroid.

\begin{tabular}{|c|c|c|c|c|c|}
\hline \multirow{2}{*}{$\begin{array}{l}\text { Number of oral steroid } \\
\text { inhaler sessions }\end{array}$} & \multicolumn{5}{|c|}{ Time between esophageal dilations, days } \\
\hline & Patient 1 & Patient 2 & Patient 3 & Patient 4 & Mean \pm SD \\
\hline 1 & 101 & 89 & 89 & 92 & $95 \pm 5.5$ \\
\hline 2 & 120 & 110 & 110 & 114 & $116 \pm 4.5$ \\
\hline 3 & 133 & 120 & 120 & 135 & $133 \pm 10.3$ \\
\hline Mean \pm SD & $118 \pm 16.1$ & $121 \pm 23.5$ & $106 \pm 15.8$ & $113 \pm 21.5$ & \\
\hline
\end{tabular}




\section{Competing interests: None}

Q. Cai ${ }^{1}$, S. S. Yarandi', R. D. Kung', J. M. Brown' ${ }^{1}$, H. Xu ${ }^{1,2}$, Q. Cai ${ }^{1}$

${ }^{1}$ Division of Digestive Diseases, Emory University School of Medicine, Atlanta, Georgia, USA

${ }^{2}$ Department of Gastroenterology, The First Bethune Hospital of Jilin University, Changchun, Jilin, China

\section{References}

1 Spechler SJ. AGA technical review on treatment of patients with dysphagia caused by benign disorders of the distal esophagus. Gastroenterology 1999; 117: 233-254

2 Wijkerslooth LRH, Vleggaar FP, Siersema PD. Endoscopic management of difficult or recurrent esophageal strictures. Am J Gastroenterol 2011; 106: 2080-2091

3 Kochhar R, Ray JD, Sriram PV et al. Intralesional steroids augment the effects of endoscopic dilation in corrosive esophageal strictures. Gastrointest Endosc 1999; 49: 509513

4 Zein NN, Greseth JM, Perrault J. Endoscopic intralesional steroid injections in the management of refractory esophageal strictures. Gastrointest Endosc 1995; 41: 596 - 598

5 Lee M, Kubik CM, Polhamus CD et al. Preliminary experience with endoscopic intralesional steroid injection therapy for refractory upper gastrointestinal strictures. Gastrointest Endosc 1995; 41: 598-601
Bibliography

DOI http://dx.doi.org/ 10.1055/s-0032-1310257

Endoscopy 2012; 44: E408-E409

(C) Georg Thieme Verlag KG

Stuttgart · New York

ISSN 0013-726X

\section{Corresponding author}

\section{Q. Cai, MD PhD}

Division of Digestive Diseases

1365 Clifton Road, B1262

Emory University School of Medicine Atlanta, GA

USA

Fax: +1-404-778-2578

qcai@emory.edu 